\title{
Les facteurs cognitifs inhérents à l'expertise du traducteur
}

\section{Cognitive Factors Inherent to the Translator Expertise}

\author{
Carine Salem Moughayar \\ Université Saint-Joseph (ETIB), Beyrouth \\ carine.salem@usj.edu.lb
}

\begin{abstract}
This study investigates the differences between novice and expert translators. Six expert translators and six translation students were asked to translate a text into Arabic and explain their translation process. The results show a difference in the translation quality, expertise of translation and the way novices and experts think and solve the translation problems. Numerous factors such as memory, emotions, decision making, problem solving, etc. co-determine successful experts. A translator expert is not just someone who has translation skills, it's also someone who understands how his knowledge is organized; he behaves automatically and fluidly without conscious control.
\end{abstract}

Keywords: translation skills, expert vs novice, expertise, emotion, memory, emotional intelligence

\section{INTRODUCTION}

Depuis les temps anciens, la traduction a été considérée comme un art, et l'on n'a pu lire des ouvrages scientifiques sur ce sujet que depuis la deuxième moitié du siècle dernier. Ces textes portent notamment sur la formation des traducteurs, les compétences que doivent acquérir les étudiants, le processus de traduction et les approches qui pourraient être utiles à l'enseignement des apprentis traducteurs. Ainsi, le métier de traducteur a beaucoup évolué; les ouvrages traitant de la 
pédagogie de la traduction se concentrent davantage sur la qualité des traductions, les relations entre enseignants et étudiants, l'apport de nouvelles technologies, les compétences stratégiques, linguistiques et extralinguistiques. Mais ce n'est qu'à partir de 1998 que diverses études ont abordé les compétences psycho-physiologiques du traducteur. C'est le cas notamment des études menées par le groupe PACTE (2005), par Göpferich (2008) et par le groupe d'experts EMT (2009, 2017) ${ }^{1}$. Nous tenterons, dans notre article, de montrer que les composantes cognitives telles que la mémoire, l'émotion, l'attention ainsi que les aspects comportementaux et les mécanismes psychomoteurs sont inhérents à l'expertise du traducteur et que les compétences purement traductionnelles ne suffisent pas pour atteindre l'expertise.

\section{LES COMPÉTENCES TRADUCTIONNELLES}

Le groupe PACTE (Proceso de Acquisición de la Competencia Traductora y Evaluación) de l'Université autonome de Barcelone, dirigé par Amparo Hurtado Albir, a pour objectif principal d'étudier les compétences en traduction. PACTE a conduit une recherche empirique-expérimentale sur la compétence traductionnelle (CT) et a défini cette dernière de la façon suivante : « La CT est le système sousjacent de connaissances déclaratives et essentiellement opérationnelles, nécessaire pour traduire. La CT possède en outre quatre particularités : (1) c'est une connaissance experte que ne possèdent pas tous les bilingues ; (2) c'est une connaissance essentiellement opérationnelle et non pas déclarative ; (3) elle est constituée de plusieurs sous-compétences interconnectées ; (4) la composante stratégique, comme pour toute connaissance opérationnelle, joue un rôle déterminant ». Ainsi, la CT distingue les traducteurs des bilingues, elle est une connaissance opérationnelle un savoir comment, difficile à verbaliser, qui s'acquiert par la pratique et qui est mis en œuvre essentiellement de manière automatique. Le groupe PACTE affirme que : « L'acquisition de la CT est un processus de reconstruction et de développement des sous-compétences de la CT et des composantes psychophysiologiques ». Le groupe de recherche n'a pas vraiment nommé en détails les compétences en traduction, mais a plutôt développé les sous-compétences que les compétences. Ces sous-compétences s'imbriquent les unes dans les autres durant l'opération traduisante. Les deux tableaux ci-dessous schématisent les sous-compétences en traduction :

\footnotetext{
${ }^{1}$ Le réseau européen des masters en traduction (European Master's in Translation network) également appelé «EMT », a publié son référentiel de compétences de traducteurs et de traduction en janvier 2009. Toutefois, suite aux évolutions technologiques et aux besoins du marché, il a été révisé et mis à jour en 2017.
} 
Tableau 1. Sous-compétences en traduction (2007)

\begin{tabular}{|l|l|l|}
\hline \multirow{2}{*}{$\begin{array}{l}\text { Connaissances } \\
\text { opérationnelles }\end{array}$} & Sous-compétence bilingue & $\begin{array}{l}\text { Connaissances pragmatiques et sociolin- } \\
\text { guistiques. }\end{array}$ \\
\cline { 2 - 3 } & Sous-compétence instrumentale & $\begin{array}{l}\text { Connaissances concernant l'utilisation } \\
\text { des sources de documentation et les tech- } \\
\text { nologies de l'information. }\end{array}$ \\
\cline { 2 - 3 } & Sous-compétence stratégique & $\begin{array}{l}\text { Connaissances concernant l'efficacité du } \\
\text { processus de traduction et la résolution } \\
\text { des problèmes rencontrés. }\end{array}$ \\
\hline \multirow{2}{*}{$\begin{array}{l}\text { Connaissances, } \\
\text { déclaratives, } \\
\text { implicites et explicites }\end{array}$} & $\begin{array}{l}\text { Sous-compétence de connaissances } \\
\text { en traduction }\end{array}$ & $\begin{array}{l}\text { Principes qui régissent la traduction et } \\
\text { les aspects professionnels. }\end{array}$ \\
\cline { 2 - 3 } & Sous-compétence extralinguistique & $\begin{array}{l}\text { Connaissances biculturelles, encyclopé- } \\
\text { diques, thématiques... }\end{array}$ \\
\hline
\end{tabular}

Pour ce qui est des composantes psychophysiologiques, elles comprennent les composantes cognitives, les aspects comportementaux et les mécanismes psychomoteurs.

Tableau 2. Composantes psychophysiologiques (2007)

\begin{tabular}{|l|l|l|l|}
\hline \multirow{2}{*}{$\begin{array}{l}\text { Composantes } \\
\text { Psychophysio- } \\
\text { logiques }\end{array}$} & Composantes cognitives & Aspects comportementaux & Mécanismes psychomoteurs \\
\cline { 2 - 4 } & $\begin{array}{l}\text { La mémoire, la perception, } \\
\text { l'attention et l'émotion. }\end{array}$ & $\begin{array}{l}\text { La curiosité intellectuelle, } \\
\text { la persévérance, la rigueur, } \\
\text { l'esprit critique, la connais- } \\
\text { sance et la confiance en soi. }\end{array}$ & $\begin{array}{l}\text { La créativité, le raisonne- } \\
\text { ment logique, l'analyse, la } \\
\text { synthèse. }\end{array}$ \\
\hline
\end{tabular}

Ainsi, les principales compétences qu'un apprenti traducteur doit acquérir durant sa formation sont les suivantes : le traducteur est, avant tout, un professionnel de la langue, ce qui signifie qu'il doit avoir une très bonne connaissance des langues qu'il maîtrise. D'ailleurs, compte tenu de l'évolution de la technologie, il est essentiel de savoir tirer profit des outils technologiques qui facilitent la tâche du traducteur. En outre, le traducteur doit posséder une connaissance du marché du travail et des aspects commerciaux du métier. Toutes ces compétences montrent la diversité et la polyvalence des tâches du traducteur et de tout ce qu'il doit connaître et maîtriser afin de produire un texte d'arrivée de qualité et d'évoluer dans le milieu professionnel. Cependant ces compétences traductionnelles ne sont pas suffisantes pour qu'un traducteur acquière le niveau d'expertise. L'expertise comprend aussi le savoir-être et se manifeste à travers le comportement, la conduite, la coopération, l'autocritique, l'auto-motivation, etc. 


\section{L'EXPERTISE PROFESSIONNELLE}

Il est vrai que la formation universitaire joue un rôle dans l'acquisition de l'expertise, qui est le plus haut niveau du professionnalisme. Néanmoins, cela ne suffit pas, même si l'enseignant de traduction et l'apprenant ciblent les méthodes et les stratégies inhérentes à la tâche. Compétences et expertise sont interdépendantes étant donné que la compétence, la pratique et l'évaluation sont les trois clés complémentaires et indispensables à l'expertise; il y a une rupture épistémologique entre la compétence et l'expertise. Il est vrai que la compétence est indispensable et qu'elle constitue la base de l'expertise, cependant elle est insuffisante pour la justifier. Le psychologue Anders Ericsson a proposé l'expertise cognitive en 1993 sous les termes «Théorie de la pratique délibérée » qui constitue un des éléments essentiels de l'acquisition d'une expertise dans n'importe quel domaine. Selon cette théorie, acquérir le niveau d'expertise nécessite d'appliquer des techniques d'entraînement dont le but délibéré est d'améliorer les performances. Il ne suffit pas de passer de très nombreuses heures à s'entraîner, il faut apprendre à organiser sa mémoire et avoir la volonté de progresser. Est-ce que toutes les personnes qui pratiquent par exemple le football deviennent forcément des experts ? Non. La majorité se «stabilise » à un niveau intermédiaire. Par contre personne ne reste novice. Un novice qui pratique régulièrement une activité progresse mais il finit par se satisfaire du niveau atteint et cesse d'avoir une pratique «délibérée » et par conséquent il cesse de progresser.

Par ailleurs, l'acquisition de l'expertise a été l'objet de nombreuses recherches en psychologie cognitive ${ }^{2}$. Le domaine de la traduction offre à la psychologie cognitive la possibilité d'explorer de nombreuses hypothèses ayant pour sujet les processus cognitifs et psycholinguistiques. L'expertise mérite alors d'être étudiée en traduction, non seulement pour en découvrir les réalités fondamentales, mais aussi pour dégager des programmes et idées d'entraînement et développer de nouveaux systèmes de traitement d'informations. Comment un traducteur expert agit-il si on le compare à un novice ou à un nouveau diplômé ? D'où l'importance de la recherche expérimentale que nous avons menée ci-après.

\section{CADRE MÉTHODOLOGIQUE DE RÉFÉRENCE}

En traduction, les facteurs cognitifs de l'expert méritent une attention particulière. Il est vrai que de nombreuses études ont été menées sur les facteurs psychophysiologiques en traduction, mais peu de travaux empiriques rendent compte de la

\footnotetext{
${ }^{2}$ La psychologie cognitive est l'étude scientifique de la cognition, elle est centrée sur le traitement de l'information, c'est-à-dire comment le cerveau perçoit, traite et emmagasine l'information.
} 
différence réelle entre un traducteur expert et un traducteur non-expert. L'objectif de cet article est de montrer que les facteurs cognitifs tels que la mémoire, l'attention, la prise de décision et l'émotion jouent un rôle prépondérant dans le développement de l'expertise.

C'est à partir de 1986 que des études empiriques sur les processus de traduction ont vu le jour. Krings (1986) a réalisé une étude sur les stratégies de consultation de dictionnaires chez des personnes qui traduisent des textes. Il déduit que le dictionnaire bilingue est plus utilisé que le dictionnaire monolingue.

Jeanne Dancette (1995) a mené une étude expérimentale des processus de compréhension en traduction. Elle constate que la compréhension n'est pas toujours le résultat d'une opération immédiate et qu'elle est améliorable et corrigible.

Par ailleurs, K. Anders Ericsson (2003), Robert Hoffman (1997) et Barbara Moser-Mercer (2008) ont mené diverses études sur les interprètes experts. Ils ont démontré que les performances, l'organisation des connaissances et la mémoire d'un interprète expert diffèrent de celles d'un novice. Cependant, aucune recherche empirique n'a été entreprise à ce sujet dans les écoles de traduction au Liban. Pour cela, afin de mieux comprendre certains processus dégagés des études réalisées auparavant et d'identifier les facteurs cognitifs et émotionnels qui distinguent un traducteur expert d'un non-expert, nous avons mené la présente recherche empirique. Nous sommes conscients des limites de notre étude vu le nombre limité de sujets qui y ont participé. Il serait toutefois intéressant de les ajouter aux analyses faites jusqu'à présent dans ce domaine. Nous avons mené une recherche expérimentale auprès d'étudiants de Master de l'École de traducteurs et d'interprètes de Beyrouth (ETIB) et de professionnels en traduction diplômés de l'ETIB. Cette recherche entreprise dans le cadre de la préparation d'une thèse de doctorat a pour but d'identifier les facteurs cognitifs inhérents à l'expertise du traducteur et de montrer comment ils influencent son travail.

\section{MÉTHODE}

\subsection{Sujets}

Nous avons demandé à six étudiants de l'École de traducteurs et d'interprètes de Beyrouth (ETIB) de traduire un texte du français vers l'arabe. Il s'agissait d'étudiants en première année de master, qui n'avaient aucune année d'expérience en traduction. Afin d'éviter une hétérogénéité au sein du groupe quant à leur niveau dans les langues source et cible, nous avons demandé à leurs enseignants de traduction français-arabe de nous indiquer six étudiants qui ont plus ou moins le même niveau.

Le deuxième groupe est composé de six traducteurs professionnels et considérés comme « experts»; ils ont tous suivi leur formation à l'ETIB avec plus de 15 ans 
d'expérience en traduction et la traduction représente la source de revenu principale pour eux. Par ailleurs, les 12 sujets sont de langue maternelle arabe et travaillent à partir du français et de l'anglais.

\subsection{Outils de recherche et déroulement}

Nous mettons en annexe le texte choisi pour cette étude. Il a été choisi d'un commun accord entre les directrices de l'ETIB et du cycle doctoral. C'est un texte onusien d'environ 350 mots en français. Nous avons accordé une heure de temps pour la traduction.

Les 12 sujets étaient tous présents dans la même salle, donc dans les mêmes conditions de travail. L'objectif de la traduction était d'évaluer les compétences traductionnelles des deux groupes. Donc la traduction comme produit. Nous avons élaboré une grille d'évaluation, que nous reproduisons en annexe, pour la correction des traductions.

Tout de suite après la traduction, les participants ont complété un questionnaire de 10 questions qui a fait l'objet d'un test pilote pour nous assurer que les questions étaient claires et cohérentes. Le questionnaire a été préparé en vue d'expliciter l'entretien. Les questions tournent autour du texte traduit, des difficultés rencontrées et de leur résolution, des étapes suivies lors de la traduction du texte et des émotions ressenties durant l'acte de traduire.

La troisième étape consistait à faire un entretien individuel avec chacun des participants. L'entretien a aussi fait l'objet d'un test pilote. Le but du questionnaire et de l'entretien est d'identifier les facteurs cognitifs qui pourraient influencer les traducteurs dans le processus de traduction. Donc la traduction comme processus. Tous les entretiens ont été enregistrés puis transcrits. Nous avons donné à chaque participant son texte de traduction et nous lui avons accordé un temps de réflexion pour le relire et revoir la correction ainsi que la grille d'évaluation. Il est à noter que la correction a été effectuée par un correcteur qui n'était pas au courant de l'appartenance des sujets à un groupe ou à l'autre. Le correcteur est un enseignant et un expert en traduction; il a effectué son évaluation en jumelant les critères de l'évaluation académique avec les critères adoptés dans les concours de traduction réalisés dans les organisations internationales.

Ensuite nous avons posé des questions concernant le texte et l'acte de traduire. L'entretien était composé de quatre parties :

- La première concerne la mémorisation : le but était de savoir si le texte était toujours disponible dans leur mémoire et de comprendre comment fonctionne la mémoire d'un expert et d'un non-expert. Par ailleurs nous avons essayé de voir si les émotions ressenties durant l'acte de traduire pouvaient jouer un rôle dans la qualité de la traduction. 
- La deuxième partie se base sur les sources d'aide auxquelles les traducteurs ont eu recours pour dépasser les obstacles et résoudre les incertitudes et comment trancher en cas de doute. Nous avons demandé des clarifications pour essayer de savoir si les traducteurs ont eu recours aux facultés associatives, c'est-à-dire aux compétences linguistiques, stratégiques, etc. déjà acquises ou à l'expérience.

- La troisième partie consiste à analyser les erreurs de traduction commises et leurs causes. Nous avons essayé de comprendre à quoi sont dues ces erreurs : au manque d'expérience, à la lenteur dans la prise de décision, aux conditions de passation du test, au doute, aux ambiguités dans le texte de départ, à une mauvaise compréhension, etc.

- La quatrième partie consiste à déterminer si la compréhension du texte de départ était accessible ainsi que les éléments qui l'ont rendue accessible ou inaccessible.

Ainsi la triangulation des outils de collecte nous a permis de valider les données auxquelles nous avons abouti dans notre recherche.

\subsection{Résultats}

Nous présentons dans le tableau 3, le nombre et le types d'erreurs commises par chacun des 12 traducteurs.

Tableau 3. Nombre et types de fautes par sujet

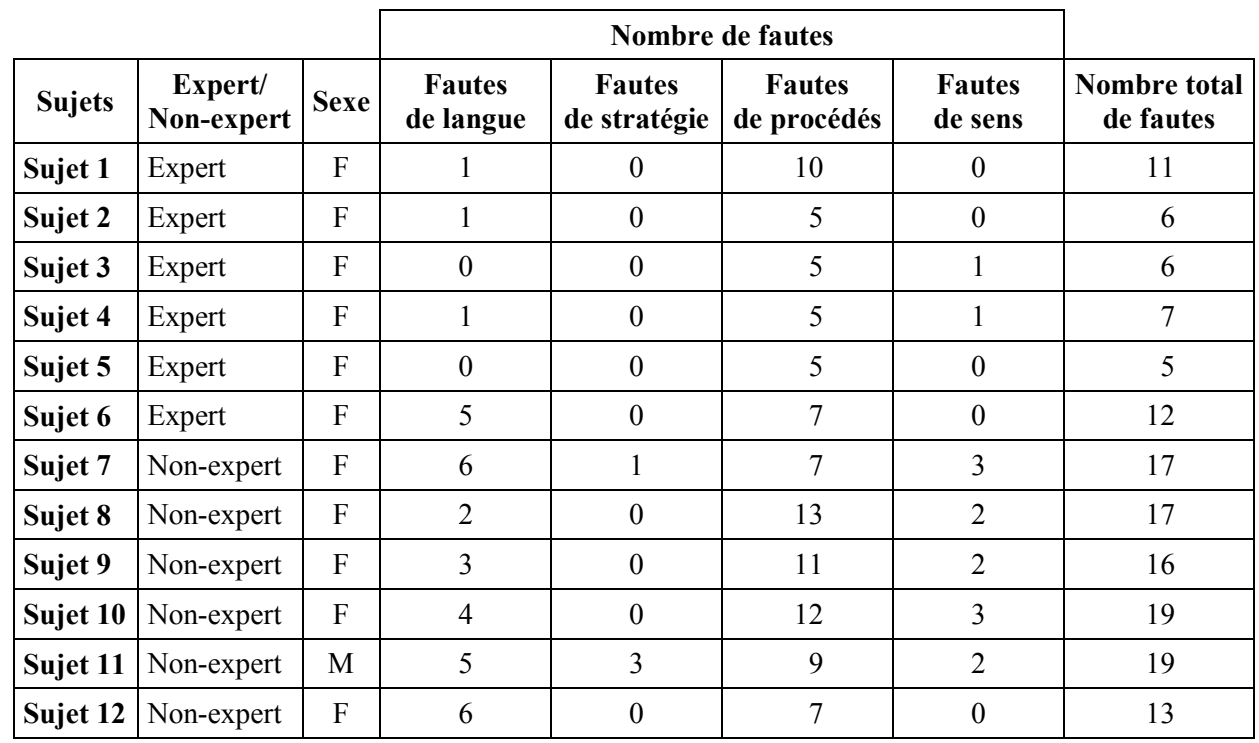


La moyenne des fautes commises par les experts et les non-experts est présentée dans le tableau 4 (par type de fautes).

Tableau 4. Moyenne des fautes par groupe de traducteurs experts et non-experts

\begin{tabular}{|l|c|c|c|}
\hline \multirow{2}{*}{\multicolumn{1}{c|}{ Type de fautes }} & \multicolumn{2}{c|}{ Moyenne des fautes } & \multirow{2}{*}{ Sig } \\
\cline { 2 - 4 } & Expert & Non-expert & \multirow{2}{*}{$\mathbf{0 , 0 1 4}$} \\
\hline Fautes de langue & 1,33 & 4,33 & 0,207 \\
\hline Stratégie & 0,00 & 0,67 & $\mathbf{0 , 0 2 1}$ \\
\hline Procédés & 6,17 & 9,83 & $\mathbf{0 , 0 0 7}$ \\
\hline Sens & 0,33 & 2,00 & $\mathbf{0}$ \\
\hline
\end{tabular}

Les valeurs de $\mathrm{Sig}<0,05$ sont marquées en gras indiquant une valeur significative entre les moyennes.

Nous avons réalisé le T-test (Test de Student) pour déterminer s'il y a une différence significative entre les experts et les non-experts. Le tableau 2 nous montre une différence réelle entre les deux groupes par rapport au type et au nombre d'erreurs commises, sauf au niveau de la stratégie employée. Les traducteurs non-experts ont commis davantage d'erreurs que les traducteurs experts. En effet, les experts ont commis en moyenne 7,8 erreurs alors que les non-experts en ont commis 16,8. Les non-experts ont commis en moyenne 4,3 fautes de langue, 2 fautes de sens et 9,8 fautes de procédés, alors que les experts en ont commis 1,3, 0,3 et 6,1 respectivement.

Dans le tableau 4, nous présentons une synthèse des résultats de l'évaluation des 12 traductions, en mettant l'accent sur la compréhension du texte source, la réexpression en arabe et l'utilisation de l'outil informatique.

Tableau 5. Nombre de traducteurs experts et non-experts qui ont montré une maîtrise des éléments suivants « Maîtrise du sujet, cohésion, cohérence, destinataire, utilisation de l'outil informatique »

\begin{tabular}{|l|c|c|c|c|c|}
\cline { 2 - 6 } \multicolumn{1}{c|}{} & \multicolumn{5}{c|}{ Nombre de traducteurs } \\
\cline { 2 - 6 } \multicolumn{1}{c|}{} & $\begin{array}{c}\text { Maitrise du } \\
\text { sujet }\end{array}$ & Cohésion & Cohérence & Destinataire & $\begin{array}{c}\text { Utilisation } \\
\text { de l'outil } \\
\text { informatique }\end{array}$ \\
\hline Experts & 6 & 5 & 5 & 6 & 5 \\
\hline Non-Experts & 3 & 4 & 0 & 2 & 5 \\
\hline
\end{tabular}

À la lecture du tableau 5, nous pouvons observer une certaine différence entre les deux groupes d'experts et non-experts. Les traducteurs experts ont compris le texte source et ont produit un texte arabe cohérent. Leur performance s'est avérée 
meilleure que celle des non-experts. Seul l'expert 6, dont le travail a été entravé par des défaillances dans l'utilisation de l'outil informatique et par les conditions de passation, a présenté une traduction de moindre qualité. Les traducteurs experts ont pu élaborer leur propre stratégie de rédaction malgré les contraintes du vouloir dire de l'auteur du texte source. La révision qui est la dernière étape de l'acte de traduction, implique une forme individuelle, subjective et unique de l'activité rédactionnelle.

Dans le tableau 6, nous présentons les résultats de l'évaluation de la qualité des traductions selon une note $/ 20$.

Tableau 6. Moyenne des notes reflétant la qualité de la traduction par groupe de traducteurs

\begin{tabular}{|c|c|c|}
\hline \multicolumn{2}{|c|}{ Moyenne des notes } & \\
\hline Expert & Non-expert & Sig \\
\hline 15,00 & 9,67 & $\mathbf{0 , 0 0 5}$ \\
\hline
\end{tabular}

Les valeurs de Sig $<0,05$ sont marquées en gras indiquant une valeur significative entre les moyennes.

Les résultats obtenus viennent confirmer le fait que les traducteurs experts ont des compétences traductionnelles plus développées que les non-experts. Leur performance en traduction a été jugée meilleure que celle des non-experts. Seul un expert a présenté une traduction de moindre qualité.

Par ailleurs, le questionnaire auquel les traducteurs ont répondu juste après la traduction du texte et les entretiens individuels qui ont eu lieu quelques jours plus tard ont révélé ce qui suit : Les non-experts ont trouvé le texte de niveau moyen. Ils ont expliqué qu'ils ont rencontré des difficultés au niveau de la terminologie et des structures. À titre d'exemple : « conseil d'administration du programme des Nations Unies pour le développement », « état des consultations », « rapport sur le développement humain », « session annuelle », « des grandes conclusions ». Ils ont également précisé que c'est un domaine nouveau pour eux et qu'ils n'ont pas beaucoup travaillé ce genre de texte dans leur parcours universitaire. Un des traducteurs nonexperts a dit : «J'ai eu des difficultés à comprendre le texte de départ. Ce sont de nouveaux concepts pour moi ; un nouveau domaine et une nouvelle terminologie ».

En réponse à la question «A quoi sont dues les erreurs? », les non-experts ont cité les facteurs suivants : le stress, l'angoisse, le manque d'expérience et le manque de temps. La plupart ont insisté sur le fait que le temps qui leur a été accordé pour réaliser la traduction n'était pas suffisant. De même, ils ont expliqué que pour résoudre les difficultés rencontrées, ils ont eu recours à des recherches dans des dictionnaires et banques de données et sur des corpus de textes.

La plupart des traducteurs non-experts ont répondu par la négative à la question « Est-ce que le texte que tu as traduit est toujours ancré dans ta mémoire ? » Ils ont 
ajouté qu'en général, les textes qu'ils traduisent ne restent pas présents dans leur mémoire ; c'est le «plaisir » qui joue un rôle important. «Tout dépend du texte, quand je sens un plaisir en traduisant ou quand j'aime le texte, je le retiens et il reste ancré dans ma mémoire. Mais quand c'est un texte ou un sujet que je n'aime pas, je le traduis par obligation donc ça passe inaperçu », a déclaré une traductrice non-experte.

La discussion avec les traducteurs non-experts a montré que la plupart d'entre eux étaient angoissés lors de la traduction; ils ont dit qu'ils n'étaient pas à l'aise et qu'ils avaient peur de ne pas terminer durant le temps imparti. «Je devais faire un travail de qualité en peu de temps, ce qui est stressant pour moi », a affirmé une traductrice non-experte.

A contrario, tous les traducteurs experts ont trouvé le texte facile, ils n'ont pas mentionné qu'ils ont rencontré des difficultés au niveau de la terminologie et des structures. Ils ont tous affirmé qu'ils avaient déjà traduit ce genre de texte et que le sujet leur était familier.

En ce qui concerne les erreurs qu'ils ont commises, ils ont expliqué qu'elles étaient dues à la fatigue et à l'inattention. À titre d'exemple, l'une des traductrices a affirmé que les erreurs de terminologie qu'elle a commises n'étaient pas dues à un manque de savoir et de connaissances mais plutôt à la fatigue.

Contrairement aux traducteurs non-experts, les experts ont affirmé qu'ils n'ont pas eu besoin de faire des recherches pour trouver les équivalents adéquats. Ils ont même affirmé qu'ils ont assez d'expérience pour être en mesure de résoudre les problèmes qu'ils pourraient rencontrer sans avoir recours aux banques de données. De plus, les experts ont affirmé qu'ils retiennent et stockent dans leur mémoire tous les textes qu'ils traduisent ainsi que les termes et les équivalents; ils les utilisent pour traduire d'autres textes. L'une des traductrices a même déclaré que chaque texte qu'elle traduit reste casé quelque part dans sa mémoire.

Contrairement aux non-experts, les experts n'ont pas ressenti d'angoisse et de stress lors de la traduction. Seule une traductrice experte a révélé avoir ressenti une certaine angoisse due à un problème technique (utilisation de l'ordinateur) qui a entravé son travail ; elle craignait ne pas avoir le temps de terminer durant le temps imparti.

\subsection{Discussion}

Ainsi, la performance des traducteurs experts était évidemment, meilleure que celle des non-experts. De plus, les données recueillies lors des entretiens ont permis de montrer que les facteurs cognitifs ont eu un impact sur l'acte de traduction et ont joué un rôle dans le cheminement vers l'expertise.

En premier lieu, les données recueillies à partir de cette recherche ont permis de montrer que la mémoire est un facteur cognitif qui distingue un expert d'un non 
expert. Ceci confirme les études théoriques et même empiriques (Moser, Ericsson, Anders, Kintsch) réalisées dans ce domaine. En effet, la mémoire du traducteur expert joue un rôle primordial lors du traitement des informations contenues dans le texte à traduire; quand un traducteur lit un texte afin de le traduire, son système oculaire saisit les informations du texte source qui passent dans son système mnésique et où elles sont traitées de façon spécifique. Une fois dans le système mnésique, ces informations sont traitées dans ce que Baddeley (1992) a appelé «la mémoire de travail », qui joue un rôle prépondérant dans l'acte traductionnel. Durant l'acte de traduction, la mémoire de travail sollicite les informations du texte ainsi que celles qui sont déjà stockées dans la mémoire à long terme. Ces dernières peuvent être linguistiques et procédurales, elles sont le résultat d'heures d'entrainement, de pratique, d'expérience et d'expertise. Si les informations stockées dans la mémoire ne sont pas suffisantes pour l'élaboration de la traduction, à ce moment-là le traducteur a recours à la recherche.

En second lieu, les résultats de cette recherche nous ont également montré que les conditions émotionnelles ont eu un impact sur le travail des traducteurs. En effet, les traducteurs experts qui n'ont pas ressenti d'angoisse ont eu une meilleure performance. Les conditions émotionnelles agréables ont favorisé les facultés cognitives, quant à celles qui sont intrinsèques, elles les ont bloquées. Par conséquent, un freinage cognitif peut être dû aux conditions émotionnelles, même chez un expert qui possède toutes les compétences traductionnelles, tel que le sujet 6 . Le processus de traduction n'est pas uniquement d'ordre rationnel. Il est vrai que toute traduction comporte une multitude de prises de décisions, cependant ces décisions ne sont pas uniquement le fruit d'un raisonnement logique ; la démarche suivie par le traducteur est orientée par des compétences émotionnelles. Les émotions sont une source de connaissances : elles nous indiquent quelles sont les informations qui doivent être considérées avec attention. Dans la théorie de Mandler (1984), l'émotion nait de la particularité d'un événement; elle se manifeste lorsqu'on est confronté à une situation inattendue et par conséquent l'attention est dirigée et focalisée sur les circonstances qui sont à l'origine de l'état émotionnel. Ainsi, il existe un apport de l'émotion à la cognition : l'émotion joue un rôle dans la gestion des ressources cognitives et notamment des ressources attentionnelles ; c'est l'émotion qui permet au système cognitif de savoir à quelles informations il doit accorder son attention. Par ailleurs, la perturbation de l'émotivité peut avoir de lourdes conséquences sur le rendement ; la cognition a besoin de l'émotion pour fonctionner correctement. Les douze sujets qui ont participé à l'expérience ont affirmé l'importance des émotions lors de l'acte traductionnel ; quand il y a une certaine empathie entre le texte et le traducteur, le rendement est meilleur. Leurs émotions ont influencé leurs performances. Par conséquent, nos émotions influent sur notre vie et sur nos performances. Selon le psychologue américain Goleman (1997, p. 109), «Dans la mesure où nos émotions bloquent ou amplifient notre capacité de penser et de planifier, d'apprendre en vue 
d'atteindre un but lointain, de résoudre des problèmes, etc., elles définissent les limites de notre aptitude à utiliser nos capacités mentales innées et décident donc de notre avenir. Et dans la mesure où nous sommes motivés par l'enthousiasme et le plaisir que nous procure ce que nous faisons, les émotions nous mènent à la réussite. C'est en ce sens que l'intelligence émotionnelle ${ }^{3}$ est une aptitude maîtresse qui influe profondément sur toutes les autres en les stimulant ou en les inhibant ». Nous savons tous que le stress peut être motivant s'il est bien maîtrisé, ou paralysant s'il est mal maîtrisé. Ceci est identique dans l'acte de traduire : soit on se laisse submerger par la difficulté, soit on apprend à se maîtriser pour la surmonter. Ainsi, l'intelligence émotionnelle qui définit la qualité de notre rendement est à la base de notre performance. Si nos états émotionnels sont négatifs (anxiété, pessimisme, manque de confiance en soi...), la performance est basse et l'échec survient. Si, par contre, nos états sont positifs (optimisme, espoir, confiance...), la performance est meilleure et le succès est plus probable. Ceci était clair chez les six sujets non experts dont le travail a été entravé par le stress et l'angoisse et par les émotions négatives ressenties lors de l'acte de traduction qui sont dues au manque de temps, au genre du texte et aux conditions de passation du test. De plus, la confiance en soi était un élément fort remarquable chez les experts : nous avons remarqué lors des entretiens que les six sujets experts ont une confiance en eux-mêmes, ils sont convaincus de leurs choix et de leurs décisions.

En troisième lieu, les résultats de cette recherche nous ont confirmé que l'exécution d'une traduction nécessite plusieurs types de prise de décisions: la compréhension du texte source requiert un raisonnement logique basé sur les compétences linguistiques, extralinguistiques, stratégiques et instrumentales ainsi que sur les connaissances thématiques acquises au préalable et emmagasinées dans la mémoire. Quant à la reformulation, elle requiert une grande manipulation de la langue cible et une décision dans les choix des termes et des équivalents. Toutes les décisions prises durant l'opération traduisante sont basées sur une analyse rationnelle ainsi que sur un environnement personnel qui dépend de nos valeurs et de nos humeurs. «Décider, c'est établir un équilibre délicat entre la puissance de l'émotion et la force de la cognition » (Berthoz, 2003, p. 307).

Enfin, cette recherche nous a montré qu'un expert en traduction est non seulement un savant, mais aussi une personne qui a beaucoup d'expérience, qui connaît ses limites et qui maîtrise à fond le domaine de traduction ; il a la capacité d'expliquer son raisonnement, de justifier ses choix, les solutions qu'il propose et les décisions qu'il prend en fonction de son expérience dans son domaine. De surcroît,

\footnotetext{
${ }^{3}$ L'intelligence émotionnelle (IE) est une forme d'intelligence qui suppose la capacité à contrôler ses sentiments et émotions et ceux des autres. La mesure de l'IE a été mise en place par le psychologue américain Daniel Goleman; elle permet de compléter le quotient intellectuel (QI) qui mesure l'intelligence d'un sujet (Goleman, 1997).
} 
la compétence d'un traducteur expert dépend d'une interaction entre ses compétences techniques et ses compétences interpersonnelles, son intelligence académique et son intelligence émotionnelle. Tout traducteur professionnel, même s'il possède une formation solide et plusieurs années d'expérience ne devient pas automatiquement et nécessairement expert. Il est vrai que les outils offerts lors de la formation aident les futurs traducteurs à développer leurs connaissances et à les réorganiser, cependant une pratique délibérée est primordiale pour atteindre une performance experte. Par ailleurs, l'expertise est pointue dans un domaine précis, elle puise ses racines dans la formation initiale, d'où l'importance de la spécialisation au niveau du master ; ceci s'est révélé dans les traductions des non-experts qui suivent le master Traducteur de conférences et dont la performance était meilleure que celle des autres non-experts.

\section{CONCLUSION}

En conclusion, la présente recherche confirme que la traduction est un processus de communication assez complexe, qui se situe au niveau linguistique mais aussi au niveau cognitif ; c'est une activité mentale qui requiert une mémorisation, une compréhension, une prise de décision et plusieurs autres facteurs cognitifs. De plus, cette recherche nous a permis de mieux cerner les facteurs cognitifs primordiaux qui influent sur l'acte de traduction et qui jouent un rôle prépondérant dans la formation d'un traducteur expert. Des recherches ultérieures devraient être menées dans ce domaine pour mieux comprendre l'impact de ces facteurs sur une population plus large et plus hétérogène. Ceci pourrait être utilisé pour intégrer dans les cursus universitaires de traduction des sessions de formation qui visent à développer ces facteurs cognitifs dans le but de permettre aux apprentis traducteurs de mieux développer leur expertise. 


\title{
TEXTE
}

\author{
Nations Unies \\ Conseil d'administration \\ Du Programme des Nations Unies \\ Pour le développement \\ et du Fonds des Nations Unies \\ pour la population
}

\section{Etat des consultations concernant le Rapport sur le développement humain}

1. Dans sa résolution 57/264, qu'elle a adoptée en janvier 2003, l'Assemblée générale invitait le Conseil d'administration du Programme des Nations unies pour le développement et du Fonds des Nations Unies pour la population à inscrire à son programme de travail annuel un point distinct relatif au Rapport sur le développement humain en vue d'améliorer le processus de consultation avec les Etats Membres concernant ce rapport ainsi que la qualité et l'exactitude du Rapport, sans compromettre l'indépendance de sa rédaction.

2. En application de cette résolution, le présent document fait le point de la situation, à l'intention du Conseil d'administration, pour examen à la session annuelle de 2010. Il passe en revue les modalités de sélection du thème du vingtième anniversaire du Rapport sur le développement humain, ainsi que toute la série de consultations et autres mesures d'interaction tenues avec les parties prenantes à travers le monde, le processus de consultations informelles du Conseil d'administrations organisé lors de l'établissement du Rapport, et quelques-unes des grandes conclusions ressortant du Rapport de 2010.

3. Faisant fond sur les résultats des consultations et de nouveaux travaux de recherche, le Rapport devrait mettre en exergue les progrès spectaculaires réalisés en matière de développement humain au cours des 40 dernières années. Le Rapport sera également l'occasion de réfléchir aux politiques et facteurs facilitateurs ou, au contraire, inhibants, ainsi qu'aux difficultés persistantes ou nouvelles. Il ressort de l'analyse qui y est faite que les inégalités, le manque d'autonomie et les vulnérabilités constituent des entraves critiques au progrès. Le Rapport proposera des améliorations à apporter au cadre du développement humain et à la manière de le mesurer. Il relèvera l'importance essentielle que revêt l'approche du développement durable énoncée dans les objectifs du Millénaire pour le développement et le programme du Millénaire en matière de développement, et aidera à articuler l'entreprise de développement au sens large, dont les objectifs du Millénaire pour le développement constituent un élément essentiel. 


\section{QUESTIONNAIRE}

1) Nombre d'années d'expérience en traduction :

2) Comment avez-vous trouvé ce texte dans l'ensemble ?
a) Facile
b) Moyen
c) Difficile
d) Très difficile

3) Avez-vous déjà traduit ce genre de texte ?
a) Oui
b) Non

4) Les difficultés rencontrées étaient au niveau :
a) Des termes
b) Des structures
c) Des termes et des structures
d) Des notions
e) Aucune difficulté

5) Comment avez-vous résolu les difficultés ?

$\begin{array}{lll}\text { a) Recherche des termes } \square & \text { b) Recherche sur des corpus de textes }\end{array}$

(Dictionnaire / Banque de données)

6) Quelles sont les étapes suivies pour traduire ce texte?

7) Quelles sont les émotions ressenties durant cet acte de traduire ? (Angoisse, peur, joie, tristesse, colère)

8) Avez-vous révisé votre traduction?
a) Oui
b) Non

9) Avez-vous terminé durant le temps imparti ?
a) Oui
b) Non

10) Comment estimez-vous votre traduction ?
a) Assez bien
b) Bien
c) Très bien $\square$
d) Excellent $\square$ 


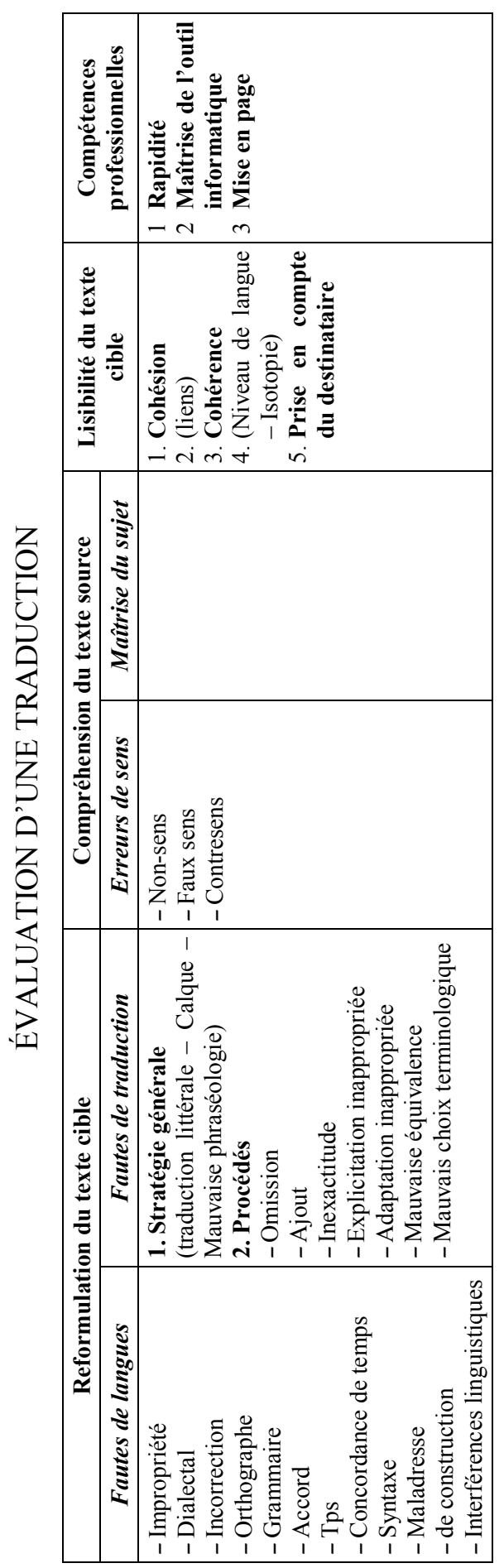




\section{RÉFÉRENCES BIBLIOGRAPHIQUES}

Baddeley, A. (1992). « Working Memory: the interface between memory and cognition ». Journal of Cognitive Neuroscience, 4, 281-288.

Berthoz, A. (2003). La Décision. Paris : Odile Jacob.

Camus, J.-F. (1996). La Psychologie cognitive de l'attention. Paris : Armand Colin.

Dancette, J. (1995). Parcours de traduction : étude expérimentale de processus de compréhension. Lille : Presses Universitaires de Lille.

EMT, GROUPE D'EXPERTS (2009). Compétences pour les traducteurs professionnels, experts en communication multilingue et multimédia. Bruxelles. Accessible sur le site http://ec.europa.eu/ info/sites/info/files/emt_competences_translators_fr.pdf. Dernière consultation le 18 juin 2016.

EMT, GROUPE D'EXPERTS (2017). Competence Framework. Accessible sur le site https://ec.europa. eu/info/sites/info/files/emt_competence_fwk_2017_en_web.pdf. Dernière consultation le 8 septembre 2018.

Ericsson, K.A. (2006). « The influence of experience and deliberate practice on the development of superior expert performance ». In K.A. Ericsson, N. Charness, P.J. Feltovich \& R.R. Hoffman (réd.), The Cambridge Handbook of Expertise and Expert Performance (pp. 683-703). Cambridge : Cambridge University Press.

Ericsson, K.A., Krampe, R.T. \& Tesch-Römer, C. (1993). « The role of deliberate practice in the acquisition of expert performance ». Psychological Review, 100, 363-406.

Goleman, D. (1997). L'intelligence émotionnelle. Paris : Éditions Robert Laffont.

Göpferich, S. (2009). « Towards a model of translation competence and its acquisition: the longitudinal study TransComp ». In S. Göpferich, A.L. Jakobsen, \& I.M. Mees (réd.), Behind the mind. Methods, models and results in translation process research (11-37). Frederiksberg : Samfundslitteratur.

Gouadec, D. (2007). Translation as a Profession. Amsterdam/Philadelphia : John Benjamin Publishing Company, coll. «Benjamin Translation Library (BTL)».

Hoffman, R. (1997). «The cognitive psychology of expertise and the domain of interpreting ». Interpreting, 1 (2), 189-230.

Hurtado Albir, A. (1999). Enseñar a traducir : metodología en la formación de traductores e intérpretes. Madrid : Edelsa.

Hurtado Albir, A. (2008). « Compétences en traduction et formation par compétences ». TTR : traduction, terminologie, rédaction, 21, 17-64. Accessible sur le site http://www.erudit.org/revue/ttr/ 2008/ v21/n1/029686ar.html.

Krings, H.P. (1986). Was in den Köpfen von Übersetzern vorgeht. Eine empirische Untersuchung zur Struktur des Übersetzungsprozesses an fortgeschrittenen Französischlernern. Tübingen : Narr.

Lasnier, F. (2000). Réussir la formation par compétences. Montréal : Guérin.

Lasnier, F. (2001). «Un modèle intégré pour l'apprentissage d'une compétence ». Pédagogie collégiale, $15(1), 28-33$.

Luminet, O. (2002). Psychologie des émotions. Bruxelles : Editions de Boeck.

Mandler, G. (1984). Mind and body: psychology of emotion and stress. New York : Norton.

Moser-Mercer, B. (2008). « Skill acquisition in interpreting. A human performance perspective ». The Interpreter and Translator Trainer, 2 (1), 1-28.

PACTE GROUP (2005). «Investigating Translation Competence: Conceptual and Methodological Issues ». Meta, 50 (2), 609-619. 